\title{
ON POLYNOMIAL EXPANSION OF MULTIVALENT FUNCTIONS
}

\author{
M. M. ELHOSH \\ Department of Mathematios \\ University of College of Wales \\ Aberystwyth, Wales \\ United Kingdom \\ (Received April 24, 1987)
}

ABSTRACT. Coefficient bounds for mean p-valent functions, whose expansion in an ellipse has a Jacobi polynomial series, are given in this paper.

KEY WORDS AND PHRASES. Univalent and multivalued functions, orthogonal polynomials. 1980 AMS SUBJECT CLASSIFICATION CODES. 30C50, 30C55.

1. INTRODUCTION.

Let $E_{0}=\left\{z=\cosh \left(s_{0}+i \tau\right), 0<\tau<2 \pi, s_{0}=\tanh ^{-1}(b / a), a>b>0\right\}$ be a fixed ellipse whose focl are \pm 1 . Let $a l s o r_{0}=a+b$ be the sum of the semi-axis of $E_{0}$. It is known (Szegö [1], Theorem 9.1.1], see also p. 245) that a function $f(z)$ which is regular in Int $\left(E_{0}\right.$ ) (this means the interior of $E_{0}$ ) has an expansion of the form

$$
f(z)=\sum_{n=0}^{\infty} a_{n} p_{n}(\alpha, \beta)(z)
$$

where here and throughout this paper $\alpha, \beta>-1$. This expansion converges locally uniformly in Int ( $\left.E_{0}\right)$. In [2] the author has given some coefficient bounds for functions mean p-valent and has an expanston in terms of Chebyshev polynomials in Int $\left(E_{0}\right)$. Such polynomials are generated by the special case $\alpha=\beta=-1 / 2$ in Jacobi polynomials. Other special cases of interest are the Legendre and the altraspherical polynomials generated by $\alpha=\beta=0$ and $\alpha=\beta$ respectively $[1, p .80-89]$.

In this paper we generalize results given in [2] to functions of the form (1.1) and mean $p$-valent in Int $\left(E_{0}\right)$. In view of $[2]$ we call $f(z)$ mean $p$-valent in Int $\left(E_{0}\right)$ if

$$
W(R, f)=(1 / \pi) \int_{0}^{R} \int_{0}^{2 \pi} n\left(\rho e^{i \phi}, f, \operatorname{Int}\left(E_{0}\right)\right) \rho d \rho d \phi<p R^{2}
$$

where $0<R<\infty$ and $n\left(p e^{i \phi}, f, \operatorname{Int}\left(E_{0}\right)\right)$ denotes the number of roots of the equation $f(z)$ $w$ in Interior $E_{0}$, multiplicity being take into account.

We first recall from [2]:

THEOREM A. Let $f(z)$ be mean $p$-valent in $\operatorname{Int}\left(E_{0}\right)$. Then for $z=\cosh (s+1 \tau), \exp (s)$ $=r$ and $1<r<r_{0}$ we have

$$
|f(z)|=0(1)\left(1-r / r_{o}\right)^{-2 p}
$$

where $0(1)$ depends on $a, b$ and $f$ only. 
THEOREM B. Let $f(z)$ be mean p-valent in $\operatorname{Int}\left(E_{0}\right)$ and $M(r, f)<C\left(1-r / r_{0}\right)^{-\gamma}$ where $c, \gamma>0$ and $M(r, f)=\max \left\{|f(z)|: z \in \operatorname{Int}\left(E_{0}\right)\right\}$. Set $z=\cosh (s+i \tau), \exp (s)=r$, $1<r<r_{0}$ and

$$
I_{1}\left(r, f^{\prime}\right)=(1 / 2 \pi) \int_{0}^{2 \pi}\left|f^{\prime}(\cosh (s+i \tau))\right||\sinh (s+i \tau)| d t .
$$

Then as $r+r_{0}$ we have

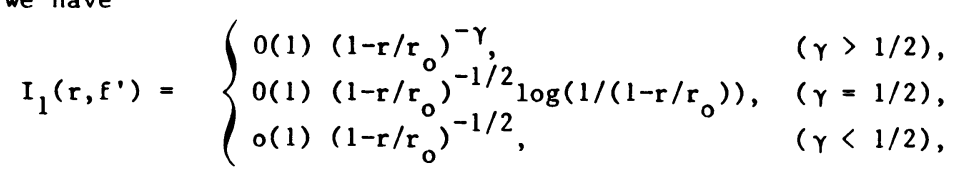

where $O(1)$ and $o(1)$ depend on $a, b, \gamma$ and $f$ only.

PROOF OF THEOREM B. Using Schwarz's inequality we have

$$
\begin{aligned}
I_{1}\left(r, f^{\prime}\right) \leq & <\left[(1 / 2 \pi) \int_{0}^{2 \pi} \mid f^{\prime}\left(\left.\cosh (s+1 \tau)\right|^{2}|f(\cosh (s+1 \tau))|^{\lambda-2}|\sinh (s+i \tau)|^{2} d^{2}\right)^{1 / 2}\right]^{1 / 2} \\
& \left.\times\left[(1 / 2 \pi) \int_{0}^{2 \pi}|f(\cosh (s+i \tau))|^{2-\lambda} d \tau\right)^{1 / 2}\right]^{1 / 2}
\end{aligned}
$$

where $0<\lambda<2$. Theorem $B$ now follows in the same way as estimating inequality (14) of [2] by using [2, Lemmas 3 and 4].

We now need a suitable coefficient formula.

LEMMA 1.1. Let $f(z)=\sum_{n=0}^{\infty} a_{n} p_{n}(\alpha, \beta)$ (z) be regular in $\operatorname{Int}\left(E_{0}\right)$ and

$E=\{z=\cosh (s+1 \tau), 0<\tau<2 \pi\}$. Then for a fixed s so that $0<s<s_{0}$ we have

$$
\begin{aligned}
a_{n} & =\left(k_{n}^{(\alpha, \beta)} / h_{n}^{(\alpha, \beta)}\right)(1 / 2 \pi i) \int_{E} \frac{f(z)}{z^{n+1}} d z, \quad(n>0), \\
\frac{1}{2}(n+\alpha+\beta+1) a_{n} & =\left(k_{n}^{(\alpha+1, \beta+1)} / h_{n-1}^{(\alpha+1, \beta+1)}\right)(1 / 2 \pi i) \int_{E} \frac{f^{\prime}(z)}{z^{n}} d z, \quad(n>1)
\end{aligned}
$$

where $k_{n}^{(\alpha, \beta)}=2^{n+\alpha+\beta+1} \Gamma(n+\alpha+1) \Gamma(n+\beta+1) / \Gamma(2 n+\alpha+\beta+2)$ and $h_{n}^{(\alpha, \beta)}=2^{\alpha+\beta+1} \Gamma(n+\alpha+1) \Gamma(n+\beta+1) /(2 n+\alpha+\beta+1) \Gamma(n+1) \Gamma(n+\alpha+\beta+1)$.

We note here, using Stirling's formula from Titmarsh [3, p. 57], that

$$
\mathrm{K}_{\mathrm{n}}(\alpha, \beta) / \mathrm{h}_{\mathrm{n}}^{(\alpha, \beta)}=0(1) \mathrm{n}^{1 / 2} / 2^{\mathrm{n}}
$$

as $n \rightarrow \infty$, where $0(1)$ depends on $\alpha, \beta$ only.

PROOF OF LEMMA. We have from $[1, p .245]$ that

$$
a_{n}=\left\{\pi i h_{n}^{(\alpha, \beta)}\right\}^{-1} \int_{E}(z-1)^{\alpha}(z+1){ }^{\beta} Q_{n}(\alpha, \beta)(z) f(z) d z
$$

where $\mathrm{n}=0,1,2, \ldots$.

We now see from [1, Theorem 4.61.2], (see also Erdelyi, Magnus, Oberhettinger and Tricomi [4, p. 171], and Freud [5, p.44] that

$$
\begin{aligned}
& (z-1)^{\alpha}(z+1)^{\beta} Q_{n}(\alpha, \beta)(z)=(1 / 2) \sum_{k=0}^{\infty} \frac{1}{z^{k+1}} \int_{-1}^{1}(1-t)^{\alpha}(1+t)^{\beta}{ }_{t} p_{n}(\alpha, \beta)(t) d t \\
& =k_{n}(\alpha, \beta) / 2 z^{n+1} \text {, }
\end{aligned}
$$


where $k_{n}^{(\alpha, \beta)}$ is as defined above. In connection with this, see the argument used in the proof of formula (4.3.3) of $[1, \mathrm{p} .67]$.

Using (1.6) in (1.5) we Immeriately deduce (1.2).

Now differentiating (1.1) we see from equation (4.21.7) of [1] that

$$
f^{\prime}(z)=\sum_{n=1}^{\infty} \frac{1}{2}(n+\alpha+\beta+1) a_{n} P_{n-1}^{(\alpha+1, \beta+1)}(z) .
$$

Again, as in the proof of (1.2), we deduce from this and [1, p. 245] for $n>1$, that

$$
\begin{aligned}
\frac{1}{2}(n+\alpha+\beta+1) a_{n} & =\left\{\pi i h_{n-1}^{(\alpha+1, \beta+1)}\right\}^{-1} \int_{E}(z-1)^{\alpha+1}(z+1)^{\beta+1} Q_{n-1}^{(\alpha+1, \beta+1)}(z) f^{\prime}(z) d z \\
& =\left(K_{n-1}^{(\alpha+1, \beta+1)} / h_{n-1}^{(\alpha+1, \beta+1)}\right)(1 / 2 \pi i) \int_{E} \frac{f^{\prime}(z)}{z^{n}} d z
\end{aligned}
$$

where we have used the equation $(z-1)^{\alpha+1}(z+1)^{\beta+1} Q_{n-1}^{(\alpha+1, \beta+1)}(z)=K_{n-1}^{(\alpha+1, \beta+1)} / 2 z^{n}$ which is deduced as in (1.6). This is equation (1.3) and the proof of the lemma is now complete.

2. MAIN THEOREM.

THEOREM 2.1. Let $f(z)=\sum_{n=0}^{\infty} a_{n} P_{n}^{(\alpha, \beta)}(z)$ be mean $p$-valent in $\operatorname{Int}\left(E_{0}\right)$ and $M(r, f)<C\left(1-r / r_{0}\right)^{-\gamma}$ where $C, \gamma>0$ and $M(r, f)$ is as defined above. Then, as $n \rightarrow \infty$ we have

$$
\left|a_{n}\right|=r_{0}^{-n} \begin{cases}0(1) n^{r-1 / 2}, & (r<1 / 2), \\ 0(1)(\log n), & (r=1 / 2), \\ 0(1), & (r<1 / 2),\end{cases}
$$

where $O(1)$ and $o(1)$ depend on $a, b, \alpha, \beta, \gamma$ and $f$ only.

PROOF OF THEOREM 2.1. From (1.3) and Theorem B we deduce, using the bounds $|\sinh (s+1 \tau)|>\sinh s,|\cosh (8+1 \tau)|<\cosh s$ and $(1.4)$, that

$$
\begin{aligned}
& \frac{1}{2}(n+\alpha+\beta+1)\left|a_{n}\right|<\left(K_{n-1}^{(\alpha+1, \beta+1)} / h_{n-1}^{(\alpha+1, \beta+1)}\left(\cosh I_{1}\left(r, f^{\prime}\right) / \sinh ^{n} s\right)\right. \\
& <\left(K_{n-1}^{(\alpha+1, \beta+1)} / h_{n-1}^{(\alpha+1, \beta+1)}\right)\left(2^{n_{1}} I_{1}\left(r, f^{\prime}\right) / r^{n}(1-1 / r)\right) \\
& \left|a_{n}\right|=r_{0}^{-n} \begin{cases}0(1) n^{r-1 / 2}, & (r>1 / 2), \\
0(1)(\log n), & (\gamma=1 / 2), \\
o(1), & (\gamma<1 / 2),\end{cases}
\end{aligned}
$$

where we have chosen $r=((n-1) / n) r_{0}$ and provided that $1-n /(n-1) r_{0}>0$. This completes the proof of Theorem 2.1. as $n \rightarrow \infty$ we have $\quad$ Let $f(z)=\sum_{n=0}^{\infty} a_{n} P_{n}^{(\alpha, \beta)}(z)$ be mean p-valent in Int (E $\left.E_{0}\right)$. Then,

$$
\left|a_{n}\right|=r_{0}^{-n} \begin{cases}0(1) n^{2 p-1 / 2}, & (p>1 / 4), \\ 0(1)(\log n), & (p=1 / 4), \\ o(1), & (p<1 / 4),\end{cases}
$$


where $O(1)$ and $o(1)$ depend $o ; a, b, \alpha, \beta, p$ and $f$ only. In view of Theorem $A$, the proof of Corollary 2.1 follows by setting $\gamma=2 p$ in Thesrem 2.1 .

COROLlaRY 2.2. Let $f(z)=\sum_{n=0}^{\infty} a_{n} p_{n}^{(\alpha, \beta)}$ (z) be univalent in $\operatorname{Int}\left(E_{0}\right)$. Then as
$+\infty$ we have $\mathrm{n} \rightarrow \infty$ we have

$$
\left|a_{n}\right|=0(1) n^{3 / 2} r_{0}^{-n}
$$

where $O(1)$ depends on $\alpha, b, \alpha, \beta$ and $f$ only.

This corollary follows upon setting $p=1$ in Corollary 2.1 .

REMARK. Using the formula (4.21.2) of [1] and the argument used in [2, Remark 2] we see by setting $z=\xi \cosh s_{0}$ where $|\xi|=\left|\cos \tau+i \tanh s_{0} \sin \tau\right|<1$ that

$$
\begin{aligned}
f\left(\xi \cosh s_{0}\right)= & \sum_{n=0}^{\infty} \frac{\Gamma(2 n+\alpha+\beta+1)}{n ! \Gamma(n+\alpha+\beta+1)} a_{n}\left(\frac{\cosh s_{0}}{2}\right)^{n}\left\{\left(\xi-1 / \cosh s_{0}\right)^{n}\right. \\
& \left.\quad+c_{1}\left(\xi-1 / \cosh s_{0}\right)^{n-1}+\ldots+c_{n} / \cosh ^{n} s_{0}\right\} \\
= & \sum_{n=0}^{\infty} \tilde{a}_{n} \hat{P}_{n}^{(\alpha, \beta)}(\xi) \\
& \tilde{P}_{n}^{(\alpha, \beta)}(\xi)=\left(\xi-1 / \cosh s_{0}\right)^{n}+c_{1}\left(\xi-1 / \cosh s_{0}\right)^{n-1}+\ldots+c_{n} / \cosh ^{n} s_{0}
\end{aligned}
$$

where

and

$$
\tilde{a}_{n}=\Gamma(2 n+\alpha+\beta+1) a_{n} \cosh ^{n} s_{0} / 2^{n} \Gamma(n+1) \Gamma(n+\alpha+\beta+1) \text {. }
$$

Using this and Stirling's formula and letting $r_{0}+\infty$ we see that Theorem 2.1 and Corollaries 2.1 and 2.2 correspond to analogous results for the unit disk (see Hayman [6]).

\section{REFERENCES}

1. SzEGÖ, G., Orthogonal polynomials, Amer. Math. Soc. Colleg. 23, (1939).

2. ELHOSH, M.M., On mean p-valent functions in an ellipse, Proc. Roy. Soc. Edinb. 92A, (1982), 1-11.

3. TITMARSH, E., The Theory of Functions, Oxford University Press (1939).

4. ERDELYI, A., MAGNUS, W., OBERHETTINGER, F. and TRICOMI, F., higher

Transcendental Functions, II, MacGraw-Hill (1953).

5. FReUd, G., Orthogonal Polynomials, Pergamon Press (1971).

6. HAYMAN, W.K., Coefficient problems for univalent functions and related function classes, J. London Math. Soc. 40 (1965), 385-406. 


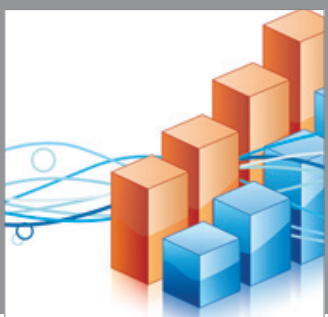

Advances in

Operations Research

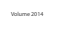

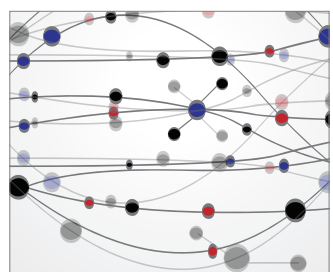

\section{The Scientific} World Journal
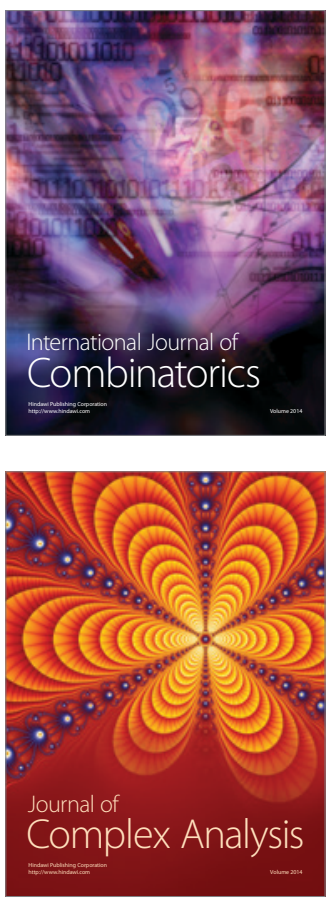

International Journal of

Mathematics and

Mathematical

Sciences
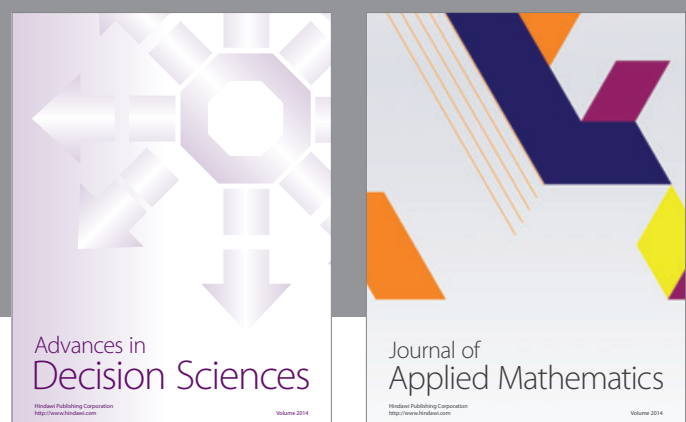

Journal of

Applied Mathematics
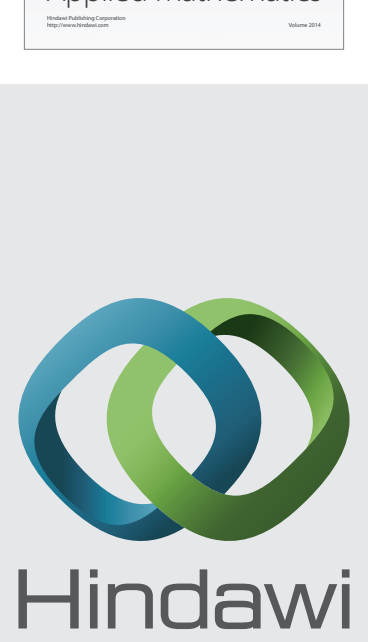

Submit your manuscripts at http://www.hindawi.com
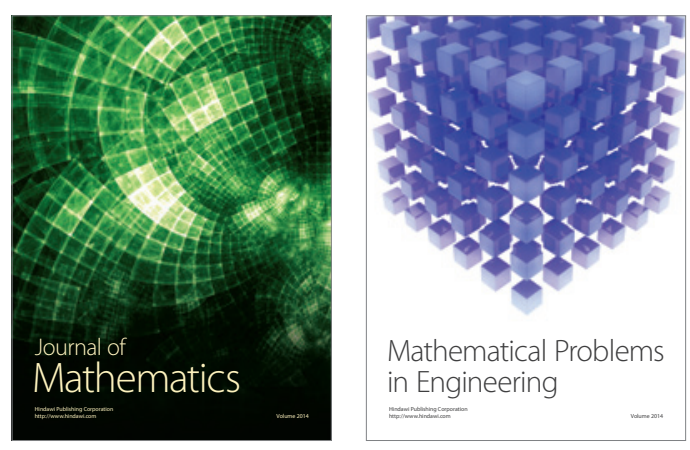

Mathematical Problems in Engineering
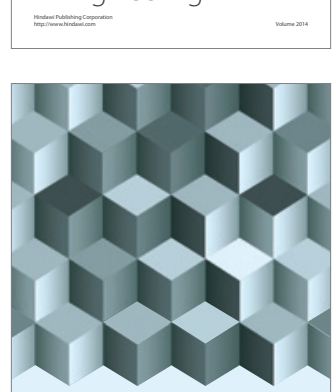

Journal of

Function Spaces
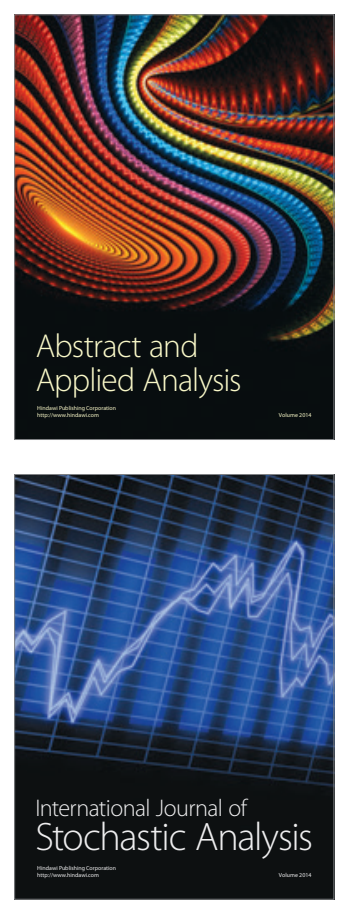

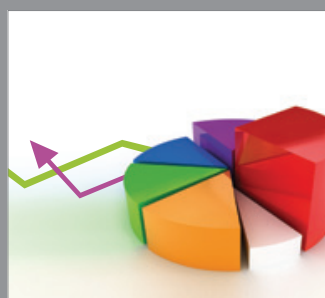

ournal of

Probability and Statistics

Promensencen
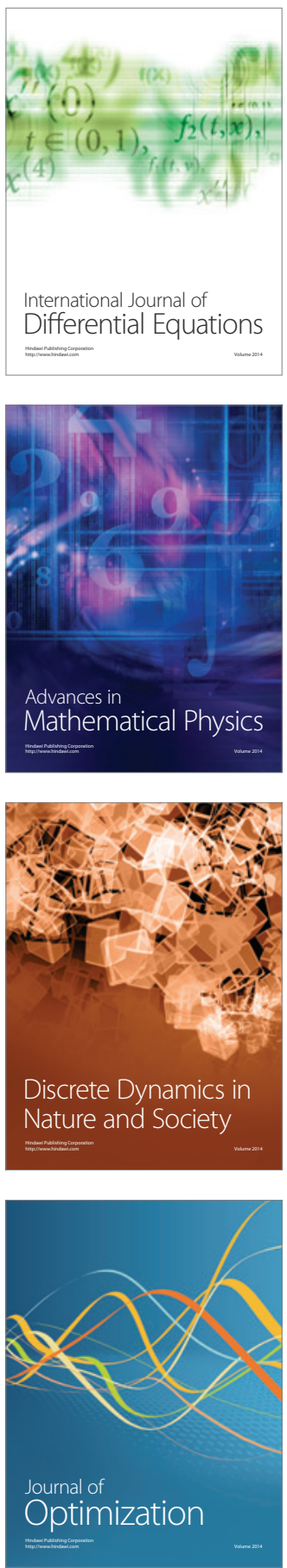